\title{
LASER INDUCED FLUORESCENCE
}

\author{
N. BRAS \\ Spectronomie des Gaz et des Plasmas, \\ Département de Recherches Physiques $\dagger$, Université Pierre et Marie Curie, \\ 4, place Jussieu, Tour 22, 75252 Paris Cedex 05, France
}

(Received 12 March, 1990; in final form 30 March, 1990)

\begin{abstract}
Laser induced fluorescence (LIF) has become a common detector of atoms and molecules allowing to determine their internal state distributions. In this paper we mention the advantages of both kinds of lasers, cw or pulsed. We review some aspects of the LIF process, such as saturation or polarization effects, which could alter the results if they were not taken into account. We also indicate how LIF can be used to measure relaxation times and thus rate constants of the relaxation processes: some experimental results obtained in our laboratory illustrate these points.
\end{abstract}

KEY WORDS: LIF, reactive collisions, intra multiplet mixing, polarization.

\section{INTRODUCTION}

Laser induced fluorescence (LIF) is a powerful technique more and more commonly used by the chemical physicist for probing gas-phase atomic or molecular species, even in minute amounts, and determining their internal states distribution.

The principle is quite simple: by absorption of one or several photons from the laser beam the probed species are pumped from an initial state $(i)$ to an excited state $(e)$ from which radiation is subsequently emitted as transitions take place to final states $(f)$. All or part of the emitted radiation is detected. Usually, each time it is possible, the light is detected at a wavelength different from the laser wavelength (i.e. the states $i$ and $f$ are different) to eliminate any spurious signal due to scattered laser light.

Since dye lasers have narrow bandwidths and are easily tunable over large wavelength domains the technique is very well adapted to determine ro-vibrational populations of reaction products. Indeed, the laser being swept, whenever it coincides with a molecular absorption line, the reaction product makes a transition to an excited electronic state from which it fluoresces. The fluorescence intensity recorded as a function of the laser wavelength is usually called an excitation spectrum. The intensities of the different lines thus observed are converted to relative populations of the various product internal states with the help of Franck Condon factors and rotational line strengths. In these excitation spectra the spectral resolution is given by the bandwidth of the laser.

\footnotetext{
† Unité Associée au CNRS nº 71.
} 
Most of the lasers employed in LIF measurements are pulsed devices, the pulse duration being of the order of a few nanoseconds. Their advantage over cw lasers is essentially related to their higher power. In particular the high peak power pulses allow frequency doubling, tripling or mixing in non linear media, crystals or gases, as well as stimulated Raman scattering, with a good efficiency. In this way sufficiently intense UV and VUV light pulses have been obtained allowing to extend the domain of investigation by LIF to more and more atoms or molecules. Presently hydrogen atoms and molecules whose band systems lie in the vacuum ultraviolet can be detected and analysed using UV, VUV or XUV radiation ${ }^{1-5,6-9}$ generated from pulsed lasers. It is more difficult to generate $\mathrm{UV}$ radiation using $\mathrm{cw}$ lasers but it is possible and has been done. ${ }^{10}$

In return pulsed lasers exhibit pulse to pulse fluctuations which make the measurement less precise than with cw lasers. Nevertheless this drawback has no or very little effect if the energy is high enough to saturate or nearly saturate the $i \rightarrow e$ transition in the first step of the LIF process. Besides the fact that it erases the undesirable effects of energy fluctuation, such a saturation increases the fluorescence signal. However in comparing laser induced fluorescence signals from different states $i$ to extract relative populations of these states it is advisable to check that saturation is achieved for all the $i \rightarrow e$ transitions. Particularly, one must be careful if the various transition strengths differ significantly or if the laser intensity varies too much through the spectral range investigated.

Another drawback of current pulsed lasers is the difficulty to make them single mode. A very narrow spectral linewidth is essential for determining, for instance, the rotational distribution of most of the molecules with accuracy. The problems due to the lack of resolution may be overcome by comparing experimental excitation spectra to spectra simulated using assumed rotational distributions. But, of course, such comparisons lead to rotational distributions much less reliable that the ones deduced from well resolved excitation spectra. However not all applications of LIF require a very narrow laser linewidth. For instance light molecules or hydrids have large rotational constant and rotational excitation spectra are obtained with a sufficient resolution even with current pulsed lasers of $\simeq 10 \mathrm{GHz}$ linewidth.

Besides these points related to energy and linewidth there is another one which necessitates attention. It concerns the polarization of the lasers. Indeed even for an isotropic sample the laser induced fluorescence has a nontrivial polarization dependence. The detected light is independent of the polarization of the incident light in the case where the detection collects the fluorescence into all polarization states and if the $M$ sublevels ( $M$ : laboratory space $z$ component of the total angular momentum) are equally populated. Otherwise it is necessary to take into account the directions and polarizations of the exciting and emitted photons when extracting relative populations from an excitation spectrum.

This problem has been considered by different authors. ${ }^{11-14}$ It has been pointed out how the orientation and alignment of reaction or photodissociation products can be deduced from polarized LIF spectroscopy and experiments have been performed. ${ }^{15-20}$ 
Usually, in the LIF experiment, one does not care too much of the radiative lifetimes of the emitting states $e$. Yet if there is a variation which is not taken into account in extracting populations from excitation spectra the results will be altered.

Finally we will mention how one can take advantage of the pulsed nature of a laser to study time evolution of the probed species and thus determine lifetimes, relaxation times and, in some cases, nascent populations of reaction products. Indeed let us assume that the atoms or molecules are created in the investigated state at some time $t_{1}$ (taken as time origin), by light excitation or collisions. The wavelength of the probe pulsed laser is kept fixed at a value corresponding to an $i \rightarrow e$ absorption line of the species while the laser is fired at the time $t_{2}$ with a variable delay $t=\left(t_{2}-t_{1}\right)$ with respect to $t_{1}$. The time integrated fluorescence intensity is recorded as a function of the delay $t$ to produce what we call a time resolved LIF measurement (TRLF). In particular this method allows to determine time evolution of ground or metastable states very easily.

Some of these aspects of LIF measurements are illustrated in the next sections where we present results obtained in our laboratory from analysis of excitation spectra or time resolved LIF. They concern essentially the collisions $\mathrm{Hg}^{*}+\mathrm{H}_{2}$ $\left(\mathrm{D}_{2}, \mathrm{HD}\right)$ where $\mathrm{Hg}^{*}$ denotes a mercury atom excited in one of the ${ }^{3} P_{J}$ states. Reactive and inelastic collisions have been studied.

\section{THE $\mathrm{Hg}\left({ }^{3} P_{1}\right)+\mathrm{H}_{2}\left(\mathrm{D}_{2}, \mathrm{HD}\right)$ REACTIVE COLLISIONS ${ }^{21,22}$}

The four following reactions were studied:

$$
\begin{aligned}
& \mathrm{Hg}\left(6^{3} P_{1}\right)+\mathrm{H}_{2} \rightarrow \mathrm{HgH}\left(X^{2} \Sigma^{+} ; v, K\right)+\mathrm{H}:\left(v=0, K=0: \Delta \mathrm{H}=-6340 \mathrm{~cm}^{-1}\right) \\
& \mathrm{Hg}\left(6^{3} P_{1}\right)+\mathrm{D}_{2} \rightarrow \operatorname{HgD}\left(X^{2} \Sigma^{+} ; v, K\right)+\mathrm{D}:\left(v=0, K=0: \Delta \mathrm{H}=-5900 \mathrm{~cm}^{-1}\right) \\
& \mathrm{Hg}\left(6^{3} P_{1}\right)+\mathrm{HD} \rightarrow \mathrm{HgH}\left(X^{2} \Sigma^{+} ; v, K\right)+\mathrm{D}:\left(v=0, K=0: \Delta \mathrm{H}=-6050 \mathrm{~cm}^{-1}\right) \\
& \mathrm{Hg}\left(6^{3} P_{1}\right)+\mathrm{HD} \rightarrow \mathrm{HgD}\left(X^{2} \Sigma^{+} ; v, K\right)+\mathrm{H}:\left(v=0, K=0: \Delta \mathrm{H}=-6240 \mathrm{~cm}^{-1}\right)
\end{aligned}
$$

A schematic diagram of the apparatus is presented in Figure 1. The reaction took place in a cell, the pressures of $\mathrm{Hg}$ and $\mathrm{H}_{2}$ were $\simeq 10^{-3}$ Torr and 0.5 Torr, respectively. The $\mathrm{HgH}$ molecules being unstable there was no consumption of the reagents except when $\mathrm{HD}$ was used. Because of the $\mathrm{Hg}+\mathrm{HD}$ reaction the HD molecules are partly broken into $\mathrm{H}$ and $\mathrm{D}$ atoms (directly or after decomposition of the $\mathrm{HgH}$ or $\mathrm{HgD}$ products) and the $\mathrm{H}$ and $\mathrm{D}$ atoms next recombine not only in $\mathrm{HD}$ but also in $\mathrm{H}_{2}$ and $\mathrm{D}_{2}$. Therefore the HD reagent gas was renewed before each recording of an excitation spectrum. The mercury atoms were excited in the ${ }^{3} P_{1}$ state by a nitrogen pumped dye laser $\left(L_{1}\right)$ which was frequency doubled by means of an angle tuned urea crystal to provide a pulse at $253.7 \mathrm{~nm}$. The $\mathrm{HgH}(\mathrm{HgD})$ molecules were probed by the laser $\left(L_{2}\right)$ induced fluorescence of the $A^{2} \Pi_{1 / 2}-X^{2} \Sigma_{1 / 2}^{+}$transition. 
All the fluorescence from the excited states, which corresponds to three rotational lines, was collected by a photomultiplier tube set at the output of a spectrograph. The photographic plate being replaced by a rectangular aperture in front of the photocathode of the photomultiplier tube, the spectrograph was used as a large band pass filter with sharp edges (FHMW $\simeq 20 \mathrm{~nm}$ for the $\mathrm{HgH}$ study, $\simeq 16 \mathrm{~nm}$ for the $\mathrm{HgD}$ study). The energy and the tuning of the pump laser $L_{1}$ were controlled by monitoring the intensity of the mercury fluorescence line at $253.7 \mathrm{~nm}$. The energy of the probe laser was also monitored.

Both excitation spectra and TRLIF measurements were analyzed. In the first case the probe laser $\left(L_{2}\right)$ was electronically delayed about 20 or $40 \mathrm{~ns}$ with respect to the pump laser $\left(L_{1}\right)$ while its wavelength was scanned through the $\mathrm{HgH}\left(A^{2} \Pi_{1 / 2}-\right.$ $\left.X^{2} \Sigma_{1 / 2}^{+}\right)$absorption bands. The probe laser linewidth was $\simeq 10 \mathrm{GHz}\left(3 \mathrm{~cm}^{-1}\right)$, which was narrow enough to get well resolved rotational spectra of $\mathrm{HgH}$ whose rotational constant is $5.4 \mathrm{~cm}^{-1}$ in the $v=0$ state.

On the contrary in the TRLIF measurements the probe laser was kept fixed at a wavelength $\lambda_{2}$ corresponding to a particular $A^{2} \Pi_{1 / 2}\left(v^{\prime}, J^{\prime}\right)-X^{2} \Sigma_{1 / 2}^{+}(v, J)$ transition $\left(J\left(J^{\prime}\right)=K\left(K^{\prime}\right) \pm 1 / 2\right)$ while the delay was electronically swept. These last measurements were essentially carried out to control the validity of the hypothesis of satured absorption used in extracting the relative populations of the $v, K$ states from the excitation spectra. Indeed, it was observed that the lines of the $Q$ branches had the same intensity as the corresponding lines of the $P$ and $R$ branches. This was due to the saturation of the absorption transition. Of course this saturation effect simplifies the analysis of the spectra since in that case the line intensities do not depend on the probe laser intensity nor on the Franck Condon and Hönl London factors. They depend only on the population of the lower state and on the statistical weights of the two states involved in the transition. Taking into account these statistical weights the relative populations of the various $v, K$ states were determined from excitation

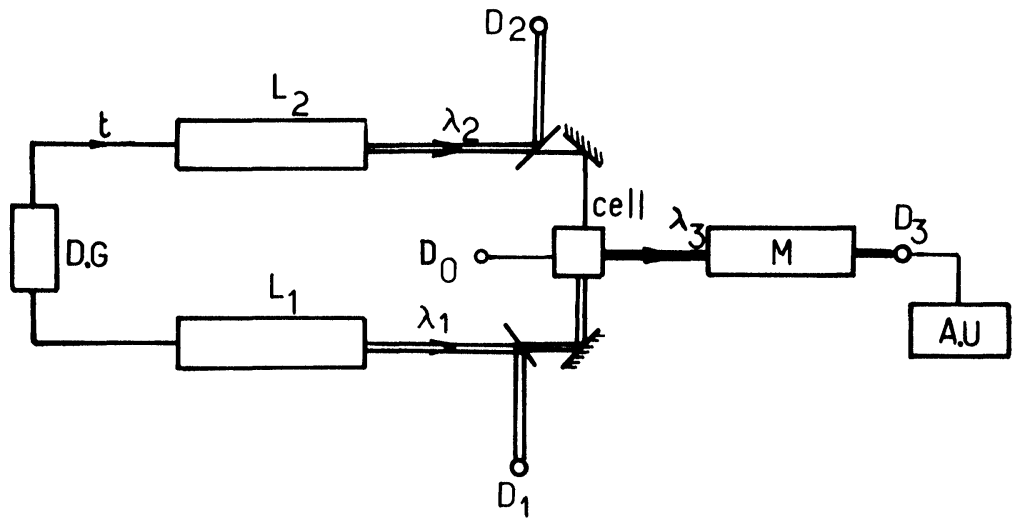

Figure 1 Schematic diagram of the apparatus. $L_{1}, L_{2}$ : pump and probe lasers; D.G.: delay generator; $D_{0}, D_{1}, D_{2}$ : detectors; $M$ : monochromator; $D_{3}$ : photomultiplier tube; A.U.: acquistion unit. 


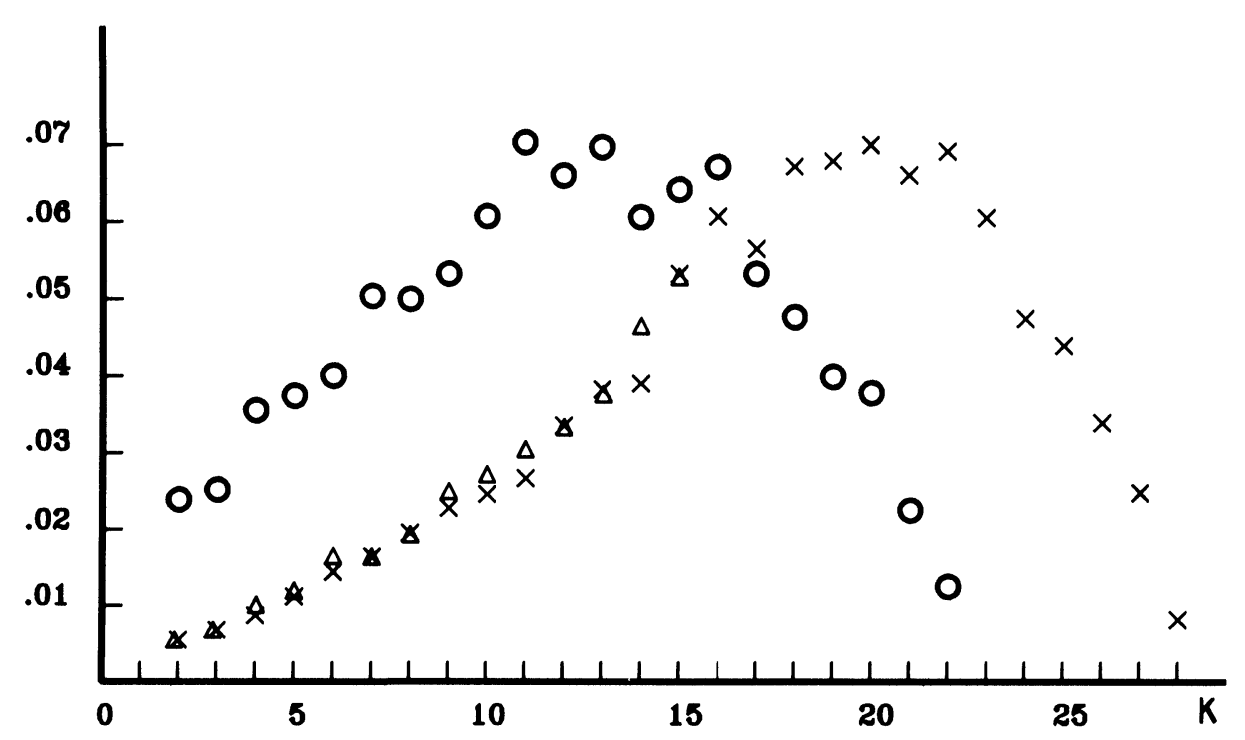

Figure 2 Initial rotational distributions of the $\mathrm{HgH}\left(X^{2} \Sigma^{+}, v=0, K\right)$ produced in the reaction $\mathrm{Hg}^{*}\left(6^{3} P_{J}\right)$ $+\mathrm{H}_{2} \rightarrow \mathrm{HgH}+\mathrm{H}$, normalized to unity: $X$ and $\Delta$ denote the results deduced from the spectral analysis and the time analysis, respectively, in the case where $\mathrm{Hg}^{*}\left(6^{3} P_{J}\right)=\mathrm{Hg}^{*}\left(6^{3} P_{1}\right) ; 0$ correspond to the case $\mathrm{Hg}^{*}\left(6^{3} P_{J}\right)=\mathrm{Hg}^{*}\left(6^{3} P_{0}\right)$.

spectra. Figure 2 presents the $K$ distribution observed in the $v=0$ states of $\mathrm{HgH}$.

TRLIF measurements were performed to check results deduced from excitation spectra. The method allows to eliminate the difficulties related to a possible detuning of the pump laser $L_{1}$ as well as those related to possible variation of the excited $v^{\prime}, K^{\prime}$ level radiative lifetimes. However the analysis is limited to the first $K$ levels $(K \leqslant 15)$. The principle consists in determining the time evolution of the $v, K$ levels by scanning the delay $t$ between the pump and probe lasers and averaging over a great number of scans. The various time evolution curves are then calibrated with the help of the Boltzmann rotational distribution which is obtained at delays sufficiently great $\left(t \geqslant 3 \mu\right.$ s for $P_{\mathrm{H}_{2}}=0.5$ Torr). When the rotational equilibrium is reached all the curves have the same time evolution, which is essentially ruled by vibrational relaxation, diffusion processes and decomposition of the molecule. Once the various curves have been calibrated, the early parts of the curves give the relative rate constants $C_{v}(K)$ of $\mathrm{HgH}(v, K)$ production. These early parts are mainly governed by the chemical reaction so that the speed of appearance of $\mathrm{HgH}(v, K)$ is essentially given by $C_{v}(K)\left[\mathrm{H}_{2}\right]\left[\mathrm{Hg}^{*}\right]_{0} \mathrm{e}^{-\mathrm{t} / \tau}, \tau$ being the lifetime of the ${ }^{3} P_{1}$ state. Thus as long as no relaxation is important the populations of the $(v, K)$ states follow laws of the form $C_{v}(K)\left[\mathrm{H}_{2}\right]\left[\mathrm{Hg}^{*}\right]_{0} \tau\left[1-\mathrm{e}^{-\mathrm{t} / \tau}\right]$ which are straight lines for $t \ll \tau$. From their slopes the relative rate constants $C_{v}(K)$ are determined. The results are displayed in Figure 2. They are in very good agreement with the ones deduced from excitation spectra. 


\section{APPLICATION OF TIME RESOLVED LIF TO MERCURY $6^{3} P_{J=0,1,2}$ RELAXATION AND INTRAMULTIPLET MIXING UNDER COLLISION WITH $\mathrm{H}_{2}, \mathrm{D}_{2}$ AND $\mathrm{HD}^{23}$}

The study was undertaken as a preliminary work to the analysis of the reactivity of $\mathrm{Hg}^{*}\left(6^{3} P_{0}\right)$ with $\mathrm{H}_{2}$. Indeed this analysis should allow to precise the mechanism of the HgH formation. ${ }^{24}$

In this experiment all the $6^{3} P_{J}$ states were optically excited to avoid any parasitic effect due, for instance, to the introduction of nitrogen, which is commonly used to populate the $6^{3} P_{0}$ state via the well-known efficient transfer $\operatorname{Hg}\left(6^{3} P_{1}\right)+\mathrm{N}_{2}(v=0)$ $\rightarrow \mathrm{Hg}\left(6^{3} P_{0}\right)+\mathrm{N}_{2}(v=0,1)$. The $6^{3} P_{0} \leftarrow 6^{1} S_{0}$ and $6^{3} P_{2} \leftarrow 6^{1} S_{0}$ transition rates are very low ( $0.18 \mathrm{~s}^{-1}$ and $0.15 \mathrm{~s}^{-1}$, respectively, in natural mercury) so that the mercury pressure was kept around 16 Torr to get sufficient signals. The $\mathrm{H}_{2}\left(\mathrm{D}_{2}, \mathrm{HD}\right)$ pressure was varied between 0.1 and 1 Torr.

The time evolutions of the three states, following the excitation of one of them, were determined by TRLIF. The probe laser was tuned at one of the $7^{3} S_{1} \leftarrow 6^{3} P_{J}$ transition wavelength and the fluorescence from the $7^{3} S_{1}$ was measured as a function of the delay between the pump and probe lasers. The probe laser was intense enough to saturate the $7^{3} S_{1} \leftarrow 6^{3} P_{J}$ transitions, which made easier and more reliable the calibration of the various time evolution curves.

The method was efficient and allowed to determine the absolute rates constants of deactivation of the three $6^{3} P_{J}$ states as well as the rate constants of the $6^{3} P_{J}^{\prime} \leftarrow 6^{3} P_{J}$ intramultiplet transfer due to $\mathrm{Hg}-\mathrm{H}_{2}\left(\mathrm{D}_{2}, \mathrm{HD}\right)$ collisions. $^{23}$

\section{THE $\mathrm{Hg}\left(6^{3} P_{0}\right)+\mathrm{H}_{2}$ REACTIVE COLLISION}

As it was mentioned by Bernier and Millié ${ }^{24}$ the comparison of the $\operatorname{HgH}(v, K)$ distributions due to the $\mathrm{Hg}\left(6^{3} P_{1}\right)+\mathrm{H}_{2}$ and $\mathrm{Hg}\left(6^{3} P_{0}\right)+\mathrm{H}_{2}$ reactions should indicate if the reactions take place adiabatically on the $\mathrm{Hg}-\mathrm{H}_{2}$ excited state surface or non adiabatically via the spin orbit coupling at the crossing of the excited and ground state surfaces.

The experiment is in progress. Excitation spectra are determined for hydrogen and mercury pressures of 0.5 or 1 Torr and $\simeq 3$ Torr, respectively. The delay between the laser exciting the $6^{3} P_{0}$ state and the one probing the $\mathrm{HgH}(v=0, \mathrm{~K})$ molecule is kept fixed at $40 \mathrm{~ns}$. First of all we have verified that at this mercury pressure the relaxation effects were still negligible at $40 \mathrm{~ns}$. This was done by comparing the $\mathrm{HgH}(v=0, \mathrm{~K})$ distributions produced in the $\mathrm{Hg}\left(6^{3} P_{1}\right)+\mathrm{H}_{2}$ reaction for various mercury pressure: for a delay of $\simeq 50 \mathrm{~ns}$ the relaxation due to mercury-mercury collisions is not discernible for mercury pressures lower than $\simeq 6$ Torr.

The first results obtained from the analysis of excitation spectra are reported in Figure 2. They show that the $\mathrm{HgH}(v=0, \mathrm{~K})$ distribution due to the $\mathrm{Hg}\left(6^{3} P_{0}\right)+\mathrm{H}_{2}$ reaction is quite different from the one obtained with $\mathrm{Hg}\left(6^{3} P_{1}\right)$.

These results are to be confirmed by analysing the time evolution of the $(v, K)$ 
states to be sure that relaxation is actually negligible. Nevertheless they indicate that the $6^{3} P_{1}$ and $6^{3} P_{0}$ states do not lead to the same $\mathrm{HgH}(v=0, K)$ distribution.

\section{POLARIZATION EFFECTS IN TRLIF MEASUREMENTS ${ }^{25}$}

Finally we would mention some polarization effects observed in TRLIF measurements. Initially the purpose of the experiment was to determine the $\operatorname{Hg}\left(6^{3} P_{1}\right)$ lifetime in pure mercury and its modification by radiation trapping.

In this study performed on monoisotopic mercury $\left({ }^{202} \mathrm{Hg}\right)$, at room temperature $\left(\mathrm{N}_{\mathrm{Hg}} \simeq 510^{13}\right.$ atoms $\left.\mathrm{cm}^{-3}\right)$, the mercury atoms were excited in the $6^{3} P_{1}$ state by a pulsed laser, at $253.7 \mathrm{~nm}$, of vertical polarization, defining the quantization axis. The probe laser at $435.8 \mathrm{~nm}\left(7^{3} S_{1} \leftarrow 6^{3} P_{1}\right)$ was fired with a variable delay and the fluorescence from the $7^{3} S_{1}$ was measured at $404.7 \mathrm{~nm}\left(7^{3} S_{1} \leftarrow 6^{3} P_{0}\right)$ as a function of this delay. The probe laser was polarized either vertically $(\pi)$ or horizontally $(\sigma)$ by means of an intracavity Rochon prism. Polarization selection $(\pi$ or $\sigma)$ of the detection was achieved by means of a Glan prism.

Due to the polarizaton of the pump laser not only population $\sigma_{0}^{0}$ but also alignment $\sigma_{0}^{2}$ were created, $\sigma_{0}^{0}$ and $\sigma_{0}^{2}$ being the components of the $6^{3} P_{1}$ density matrix, $\sigma$, developed on a set of irreducible tensor operators. These components decay at rates $\gamma_{0}$ and $\gamma_{2}$, which may be different.

According to the arrangement of both the probe laser and the detection polarizations different linear combinations of $\sigma_{0}^{0}$ and $\sigma_{0}^{2}$ may be detected resulting in quite different evolution curves. With a little algebra one can derive the evolution laws for the various polarization sets experimentally used: $I_{\pi \pi}=0, I_{\pi \sigma}$ or $I_{\sigma \pi} \propto \exp \left(-\gamma_{0} t\right)-$ $\exp \left(-\gamma_{2} t\right), I_{\sigma \sigma} \propto \exp \left(-\gamma_{0} t\right)+2 \exp \left(-\gamma_{2} t\right)$, the indexes referring to the probe and detection polarizations. Agreement between observed and calculated curve shapes was very good. The $\gamma_{0}$ and $\gamma_{2}$ constant were determined. ${ }^{25}$

This rather simple experiment clearly exemplifies the effects of polarization in LIF measurements. For instance in the $\pi \pi$ arrangement no significant LIF signal could be measured at any time.

Finally it is worth noting that the above mentioned aspects of LIF related to linewidth, saturation, polarization are met in the resonance enhanced multiphoton ionization (REMPI), which is an even more sensitive technique than LIF for the detection of atoms or molecules.

\section{References}

1. R. P. Lucht, J. T. Salmon, G. B. King, D. W. Sweeney and N. M. Laurendeau, Optics Letters 8, 365 (1981).

2. K. Tsukiyama, B. Katz and R. Bersohm, J. Chem. Phys. 82, 2889 (1985); J. Chem. Phys. 84, 1934 (1986).

3. J. E. M. Goldsmith, Optics Letters 11, 416 (1986).

4. L. Cabaret, C. Deloart and C. Blondel, Optics Communications 61, 116 (1987).

5. P. Verkek, M. Pinard, F. Biraben and G. Grynberg, Optics Communications 72, 202 (1989).

6. D. J. Kliger, J. Bokor and C. K. Rhodes, Phys. Rev. A21, 607 (1980).

7. E. E. Marinero, C. T. Rettner, R. N. Zare and A. H. Kung, Chem. Phys. Letters 95, 486 (1983).

8. W. Meier, H. Rottke, H. Zacharias and K. H. Welge, Phys. Chem. 89, 313 (1985). 
9. W. Meier, G. Ahlers and H. Zacharias, J. Chem. Phys. 85, 2599 (1986).

10. M. G. Boshier, P. E. G. Baird, C. J. Foot, E. A. Hinds, M. D. Plimmer, D. N. Stacey, J. B. Swan, D. A. Tate, D. M. Warrington and G. K. Woodgate, Phys. Rev. A40, 6169 (1989).

11. D. A. Case, G. M. McClelland and D. R. Herschbach, Mol. Phys. 35, 541 (1978).

12. Ch. H. Green and R. N. Zare, J. Chem. Phys. 78, 6741 (1983).

13. R. Altkorn and R. N. Zare, Ann. Rev. Phys. Chem. 35, 265 (1984).

14. A. J. Bain and A. J. McCaffery, J. Chem. Phys. 83, 2627, 2632, 2641 (1985).

15. M. G. Prisant, Ch. T. Rettner and R. N. Zare, J. Chem. Phys. 75, 2222 (1981).

16. P. Andresen and E. W. Rothe, J. Chem. Phys. 78, 989 (1983).

17. P. Andresen, G. S. Ondrey, B. Titze and E. W. Rothe, J. Chem. Phys. 80, 2548 (1984).

18. P. M. Doherty and D. R. Crosley, Applied Optics 23, 713 (1984).

19. F. Engelke and K. H. Meiwes-Broer, Z. Phys. A320, 39 (1985); Chem. Phys. Lett. 108, 132 (1984).

20. K. Kleinermanns and E. Linneback, Appl. Phys. B36, 203 (1985).

21. N. Bras, J. Butaux, J. C. Jeannet and D. Perrin, J. Chem. Phys. 85, 280 (1986).

22. N. Bras, J. C. Jeannet and D. Perrin, J. Chem. Phys. 87, 219 (1987).

23. N. Bras, A. El Afghani, J. Butaux, J. C. Jeannet and D. Perrin, J. Chem. Phys. 89, 1469 (1988); 91, 4142 (1989).

24. A. Bernier and P. Millié, Chem. Phys. 106, 195 (1987); A. Bernier Thesis, Université de Paris-Sud (1987).

25. N. Bras, J. Ph. Brunet, J. Butaux, J. C. Jeannet and D. Perrin, J. Phys. B17, 65 (1984). 